\title{
Correction to: Morphological reflections of evolutionary adaptations in the tongue of the white-headed duck
}

\author{
Ghasem Akbari ${ }^{1} \cdot$ Belal Hassanzadeh ${ }^{2} \cdot$ Mohammad Sadegh Madadi $^{3} \cdot$ Mohammad Babaei $^{4} \mathbb{D}$
}

Published online: 19 May 2018

(c) Japanese Association of Anatomists 2018

\section{Correction to: Anatomical Science International https://doi.org/10.1007/s12565-018-0438-x}

The affiliation of the third author has been incorrectly published in the original publication of the article. The correct affiliation is provided in this erratum.

The original article can be found online at https://doi.org/10.1007/ s12565-018-0438-x.

\section{Mohammad Babaei}

babaeimohammad@ut.ac.ir

1 Department of Basic Sciences, Faculty of Veterinary Medicine, University of Tabriz, Tabriz, Iran

2 Central Laboratory, Faculty of Veterinary Medicine, University of Tabriz, Tabriz, Iran

3 Department of Clinical Sciences, Faculty of Veterinary Medicine, University of Tabriz, Tabriz, Iran

4 Department of Basic Sciences, Faculty of Veterinary Medicine, University of Tehran, Tehran, Iran 